\title{
Diagnostics, measurement and control of electrical parameters supercapacitors
}

\author{
Juliy Boiko ${ }^{1 *}$, Tomasz Kałaczyński ${ }^{2}$ \\ ${ }^{1}$ Faculty of Information Technologies in Khmelnytskyi National University, Department of \\ Telecommunications, Media and Intelligent Technologies, 11, Instytuts'ka str., Khmelnytskyi, \\ 29016, Ukraine \\ ${ }^{2}$ Bydgoszcz University of Life Sciences and Technology, Faculty of Mechanical Engineering, \\ Kaliskiego Street 7, 85-796 Bydgoszcz, Poland
}

\begin{abstract}
This article considers possible approaches to diagnostics and measurement electrical parameters of supercapacitors. The analysis the results of diagnostics and measurement the parameters of supercapacitors has determined a significant frequency dependence of theirs. The discrepancy between the two-element equivalent circuit of supercapacitors has been established. Diagnostics and control electrical parameters of supercapacitors in the infralow frequency range can be performed by direct conversion methods. In this case, high-precision low-resistance resistors can be used as an exemplary measure. For the diagnostics and design of meters for the electrical parameters of supercapacitors of capacitors, the authors propose an iterative-recursive method for determining the numerical values of the electrical parameters of the equivalent circuit of such objects. The essence of the proposed iterative-recursive method is that at the first stage, the iterative approximation determines the number of $n$-absorption links, which it is enough to know when developing, manufacturing and operating supercapacitors. Theoretical and experimental studies of the equivalent circuit of supercapacitors show that the equivalent resistance of such capacitors and their capacitance increase with decreasing frequency of the measuring signal, and the nature of these dependences is determined by the number of $n$-absorption links and the numerical values of the elements of each link.
\end{abstract}

\section{Introduction}

Over the past decades, abroad and in Ukraine, elements of a new generation are rapidly being created and mass-produced - ultra-large capacitors $[1,2]$. The fundamental difference of such components from traditional capacitors is that they do not contain a dielectric, and their super-high electrical capacity is provided by an electric double layer (EDL), which is formed at the interface between the electrode and the electrolyte. Therefore, in the literature [1-11], such objects are also called capacitive storage, capacitors with an electric double layer, ion capacitors, as well as supercapacitors.

* Corresponding author: boiko julius@ukr.net 
Capacitors based on EDL are characterized by an ultra-large specific capacity, which is thousands of times higher than the capacity of conventional capacitors, but individual elements have rather low voltages, therefore they are connected into batteries $[4,5]$. In addition, the characteristic supercapacitors are a large value of the dielectric loss tangent $(\operatorname{tg} \delta=10 \ldots 100$ units or more) compared to conventional capacitors, because the capacitive resistance of such capacitors at frequencies of $50 \mathrm{~Hz}$ and $100 \mathrm{~Hz}$ is significantly smaller than their active internal resistance $\left(X_{\mathrm{C}}<<R\right)$ [5].

The main directions of using such capacitors are: backup power supplies at low current consumption during an emergency disconnection from the power supply network; capacitive filters for voltage and current stabilizers; pulse energy storage devices, which are capable of giving off electrical energy in the form of high-amplitude current pulses in a short period of time.

Despite extensive research and application of supercapacitors, during measurements and diagnostics of the parameters of such components, in most cases, a double-element equivalent circuit is used $[6,7]$. In fact, as experimental studies show, the equivalent circuit of such capacitors is much more complicated [8]. This simplification leads to significant errors in measuring their capacitance, and therefore complicates the control and diagnostics of their electrical parameters.

In addition, to measure the capacitances of such components, standard measuring instruments are often used to measure the capacitances of conventional capacitors. In conventional capacitors, the resistive component of the resistance is significantly less than the reactive component at measuring frequencies of $50 \mathrm{~Hz}$ and $100 \mathrm{~Hz}$. In this case, the significant difference between supercapacitors and conventional capacitors is ignored; therefore, the obtained values are characterized by a significant error and are of an estimate nature.

The analysis of the methods of measurement, control and diagnostics of capacities can be concluded that the best in relation to the measurement of the parameters of supercapacitors, there are methods of direct conversion [12-15]. Equilibration methods are reasonably highprecision and stable, but their application is limited by the lack of exemplary ultra-large capacity measures.

Among the direct conversion methods for measuring the parameters of supercapacitors, further research should be focused on the development of new modifications of the voltmeterammeter method (namely, the vector voltmeter method), as well as new modifications of the charge-discharge method (combined methods when used as a voltage generator and a current generator) [13].

Only a combination of measurement methods, using sinusoidal and even non-sinusoidal measuring signals, will make it possible to diagnose and measure with high accuracy the parameters of a complex equivalent circuit of supercapacitors that are of interest to the consumer.

So, the problem of developing specialized methods for measuring, diagnosing and controlling the parameters of supercapacitors, which would take into account the features of such components and be characterized by minimal errors, arose sharply. Only the creation of new methods or a significant improvement of existing methods for measuring ultra-large capacities will make it possible to create measuring instruments for supercapacitors, characterized by minimal measurement errors.

The article describes specialized methods for diagnostics and control parameters of supercapacitors, which take into account the features of such components and are characterized by minimal errors. Creation new methods or significant improvement of existing methods for measuring ultra-large capacities will make it possible to create measuring and control devices for supercapacitors, characterized by minimal measurement errors. 


\section{Analysis of impedance measurement approaches}

The complexity of supercapacitors lies in the fact that, in addition to the main capacitance and series active resistance, capacitances and active absorption resistances are observed in such objects [5]. Therefore, it is unacceptable to depict objects with an extra-large capacity of a simple two-element equivalent circuit [6]. This is confirmed by the experimental dependences of the capacitance and the dielectric loss tangent on the frequency of the measurement signal in Fig. 1.

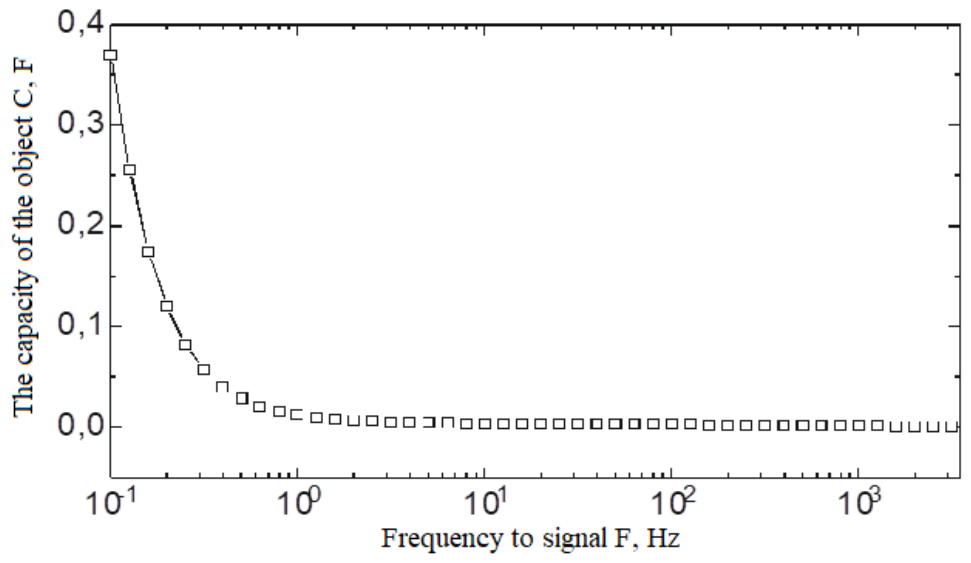

a

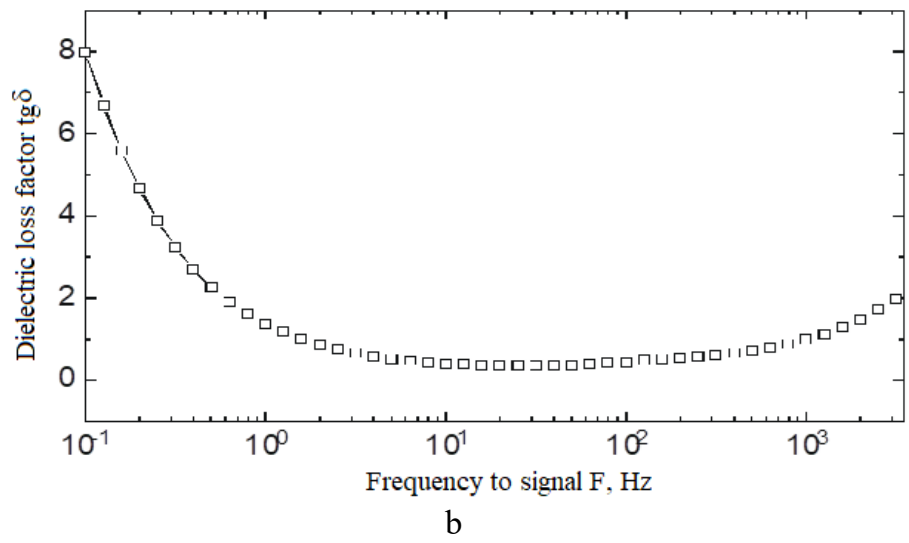

Fig. 1. Frequency dependence of capacitance (a) and dielectric loss tangent (factor) (b).

Analysis of the graphs of dependencies, which are shown in (Fig. 1), shows that the diagnostic object is a complex frequency-dependent system.

The presence of precisely the capacitances and active absorption resistances leads to significant differences between the capacitance values at the measuring frequencies of $50 \mathrm{~Hz}$ and $100 \mathrm{~Hz}$, in comparison with the capacitance values in the region of infra-low frequencies. This difference is especially noticeable when measured by the charge-discharge method [37].

So, in addition to the main capacitance of the object of measurement, it is also necessary to determine and normalize all the parameters of the multielement equivalent circuit of supercapacitors (capacitance and active absorption resistances), which are important characteristics of such components [8].

Let us consider the main approaches to measuring the parameters of complex resistances in order to form measuring problems in determining the values of the parameters of 
supercapacitors. In accordance with Ohm's law, the complex resistance $Z$ of the object of measurement (impedance) is defined as the ratio of two complex quantities: the complex voltage $\dot{U}$, which is applied to the object of measurement, and the complex current $\dot{I}$ passing through the object as a result of the action of the complex voltage $\dot{U}$.

So, in order to measure the complex resistance of a supercapacitor, represented by a simple two-element equivalent circuit, it is necessary to measure the independent components of the complex resistance: the real (active resistance $R$ ) and imaginary (reactance $X_{\mathrm{C})}$ components:

$$
\operatorname{Re} \underline{Z}=\operatorname{Re} \frac{\dot{U}}{\dot{I}}=R ; \operatorname{Im} \underline{Z}=\operatorname{Im} \frac{\dot{U}}{\dot{I}}=X_{C}
$$

From the system of two equations connecting the measured complex quantities and with the parameters of the complex resistance, the capacitance $C$ and the equivalent reactance $R$ of the supercapacitor are determined. Most of the known meters of complex resistance parameters are based on this principle $[5,13]$, and measurements can be carried out in analog or digital form. When calculating the parameters of the complex resistance based on the results of measuring the complex values of voltage and current, the accuracy of determining the desired parameters depends on the errors in measuring the complex values, which are measured by the direct method, as well as the features of the microprocessor (MP).

A characteristic feature of modern high-precision microprocessor-based meters for complex resistance parameters is the measurement of the complex values of the voltage $U$ at the measurement object and the complex current $\dot{I}$ that passes through the measurement object using one measuring channel. In these devices, the magnitude of the complex current $\dot{I}$ is measured using a reference resistor $R_{0}$, through which the measured complex current passes, creating a voltage drop $U_{0}$ across it (Fig.2).

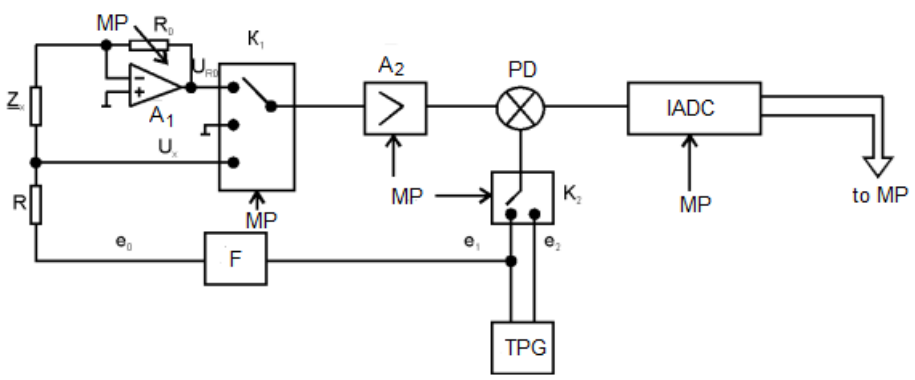

Fig. 2. Measuring range of RLC meters.

For accurate measurements, it is necessary to use only one measuring channel Fig. 2, which alternately measures the complex voltages on the object of measurement and the exemplary active resistance $R_{0}$. In this case, the measurement accuracy is significantly increased in comparison with two-channel meters, which are characterized by significant errors due to the non-identical characteristics of the measuring channels.

The measuring circle shown in (Fig. 2) consists of an unknown complex resistance $Z_{\mathrm{x}}$ and an exemplary active resistance $R_{0}$. By changing the value of $R_{0}$, the measurement range is selected. The electrical circuit is powered by an alternating voltage source based on a twophase pulse generator (TPG). This generator generates two square wave trains $e_{1}$ and $e_{2}$. 
The pulse generator is made entirely on logical elements without the use of precise analog elements, which makes it possible to obtain a phase shift between $\mathrm{e}_{1}$ and $\mathrm{e}_{2}$ with high accuracy. A sinusoidal voltage $e_{0}$ is formed from one output voltage of the TPG using a filter $\mathrm{F}$, which is fed to $Z_{\mathrm{x}}$. For this scheme, $Z_{\mathrm{x}}$ is equal to:

$$
\underline{Z}_{X}=R_{0} \frac{\dot{U}_{x}}{\dot{U}_{R 0}}
$$

Therefore, to determine the parameters $Z_{\mathrm{x}}$ with a known $R_{0}$, it is necessary to measure two complex voltages $U_{\mathrm{x}}$ and $U_{R 0}$, for example, measure their components relative to any third voltage, the phase of which will be considered zero. In this case, the measurement is carried out relative to $\mathrm{e}_{1}$.

The measurement process consists of five steps. At the first cycle, the switch $\mathrm{K}_{1}$ through the amplifier $\mathrm{A}_{2}$ connects the voltage $U_{\mathrm{x}}$ to the input of the phase detector (PD), to the reference input of which voltage $e_{1}$ is applied through the switch $K_{2}$. This voltage with the help of an integrating analog-to-digital converter (IADC) is converted into $\mathrm{a}_{1}$ code, which is stored in the memory of the MP.

On the second clock cycle, the key $\mathrm{K}_{2}$ supplies a voltage $\mathrm{e}_{2}$ to the PD input, which has a phase shift relative to the voltage $\mathrm{e}_{1}$, and the measurement and storage of the quadrature component $U_{\mathrm{x}}\left(\mathrm{a}_{2}\right)$ is carried out.

On the third and fourth clock cycles, the in-phase $\left(a_{3}\right)$ and quadrature $\left(a_{4}\right)$ components of the voltage $U_{\mathrm{R} 0}$ are measured.

In the fifth cycle, the key $\mathrm{K}_{1}$ grounding the input and the zero drift of the PD and IADC is measured ( $\left.\mathrm{a}_{5}\right)$. Based on the results of these measurements $\left(\mathrm{a}_{1} \ldots \mathrm{a}_{5}\right)$, the value of $R_{0}$ and the supply frequency of the measuring circuit, it is possible to calculate the parameters of the complex resistance for the supercapacitor using the following formulas:

Active resistance $R_{\mathrm{x}}$ :

$$
R_{X}=R_{0} \frac{\left(a_{1}-a_{5}\right)\left(a_{3}-a_{5}\right)+\left(a_{2}-a_{5}\right)\left(a_{4}-a_{5}\right)}{\left(a_{3}-a_{5}\right)^{2}+\left(a_{4}-a_{5}\right)^{2}}
$$

Reactance:

$$
X_{c}=R_{0} \frac{\left(a_{2}-a_{5}\right)\left(a_{3}-a_{5}\right)-\left(a_{1}-a_{5}\right)\left(a_{1}-a_{3}\right)}{\left(a_{3}-a_{5}\right)^{2}+\left(a_{4}-a_{5}\right)^{2}}
$$

Object capacity:

$$
C_{X}=-\frac{1}{\omega X_{C}}
$$

Dielectric loss angle tangent:

$$
\operatorname{tg} \delta=\frac{R_{X}}{\left|X_{c}\right|}
$$


Thus, the approaches to the construction of meters for the parameters of complex quantities based on the physical model of the measurement object [5] allow formulating the requirements for such meters: the method and equipment for measurement should ensure the measurement of the parameters of a multielement equivalent circuit for capacitors with an extra-large capacity; the measurement method should contain tools and means for reducing the operating frequency of the measuring signal towards low and infra-low frequencies when measuring the parameters of supercapacitors; the measuring equipment should be able to expand the range of measured capacitance values up to $1000 \mathrm{~F}$ and the dielectric loss tangent up to 100 units or more when measuring the parameters of ultra-large capacitors at infra-low frequencies; the operation of the meter should be based on a digital method for recording errors and calibrating infra-low-frequency equipment for measuring the parameters of a multielement equivalent circuit for supercapacitors.

\section{Method for determining the electrical parameters of supercapacitors}

The carried out theoretical and experimental studies of the frequency dependences of the equivalent capacitance $C(\omega)$ and active resistance $R(\omega)$ (Fig. 1) show that the determination of the parameters of the elements of the equivalent circuit of supercapacitors is a difficult measurement problem $[10,11]$. The complexity of the problem lies in the fact that the number $n$ of absorption links $R_{i} C_{i}$ for real supercapacitors is a priori unknown and different.

The general method for determining the numerical values of the equivalents of equivalent circuits of multi-element two-port can be presented as follows: if the number of elements of a multi-element $R C$ passive two-port is fixed and known, then its complex resistance can be written in the following formula:

$$
Z(p)=\frac{A_{n} p^{n}+A_{n-1} p^{n-1}+\ldots+A_{1} p+A_{0} p^{0}}{A_{2 n} p^{2 n}+A_{2 n-1} p^{2 n-1}+\ldots+A_{n+1} p^{n+1}}
$$

Where $n$ is the number of independent two-port circuits; $p=j \omega$ is the complex frequency; $A$ is a proportionality coefficient.

To determine the components of the vector of parameters of a multi-element two-port, we use discrete values of the complex resistance of its equivalent circuit:

$$
Z_{i}(p)=R_{i}+j X_{i}, \quad(i=1,2, \ldots, n)
$$

Where $R_{i}$ is the measured value of the active component of the resistance; $X_{i}$ is the measured value of the reactive component of the resistance.

$$
Z_{i}\left(j \omega_{i}\right)=R_{i}+j X_{i}, \quad(i=1,2, \ldots, n) .
$$

By expression (7), we obtain an inhomogeneous system of equations for $A_{k}$ for the number of frequencies $L$.

$$
\left\{\begin{array}{c}
A_{n} p^{n}+A_{n-1} p^{n-1}+\ldots+A_{\kappa} p^{\kappa}+\ldots A_{1} p^{1}+A_{0}=0 \\
A_{2 n} p^{2 n}+A_{2 n-1} p^{2 n-1}+\ldots+A_{2 \kappa} p^{2 \kappa}+\ldots+A_{n+1} p^{n+1}=0
\end{array}\right.
$$

Where $\kappa=1,2, \ldots, n$.

In vector form, system (10) has the form: 


$$
\bar{P} \bar{A}=0
$$

To determine $A_{k}(k=1,2, \ldots, 2 n)$ with (11), it is necessary to measure the value of the complex resistance at least $n$ different frequencies. Then $\operatorname{det} P \neq 0$, hence all $A_{k}$ are uniquely determined.

After solving system (11), the following system of nonlinear equations is solved:

$$
\left\{\begin{array}{c}
A_{1}=f_{1}\left(R_{1}, X_{1}\right) \\
A_{2}=f_{2}\left(R_{2}, X_{2}\right) \\
A_{2 n}=f_{2 n}\left(R_{2 n}, X_{2 n}\right)
\end{array}\right.
$$

In vector form, the system (12) has the form:

$$
\bar{F}(\bar{R}, \bar{X})=\bar{A},
$$

Where the left-hand sides of the equations of the system are the sums of products of different degrees of the sought parameters.

This technique allows you to determine all the parameters of a multi-element two-port, but requires a known number $n$ of independent two- port circuits.

For the equivalent circuit of supercapacitors, the number of $R_{i} C_{i}$ absorption units, and hence the number $\mathrm{n}$ of independent circuits for real capacitors, is a priori unknown and different.

For the development of meters for the electrical parameters of supercapacitors, the authors propose an iterative-recursive method [5] for determining the numerical values of the electrical parameters of the equivalent circuit of such objects.

The essence of the proposed iterative-recursive method is that at the first stage, the iterative approximation determines the number of $n$-units of absorption $R_{i} C_{i}$, which it is enough to know when developing, manufacturing, operating and diagnosing supercapacitors.

At the second stage, using recursive calculations based on the measured values of the equivalent capacitance and active resistance, the numerical values of the electrical parameters $r, C_{0}, C_{i}, R_{i}$, and $R_{0}$ of the supercapacitor equivalent circuit are determined.

At the first stage, the number of $n$-links of $R_{i} C_{i}$, absorption is determined according to the following criteria:

- frequency range $f_{\min } \ldots f_{\max }$, in which supercapacitors are used;

- frequency sampling step $\Delta f$, which is determined by the rate of change of the frequency response;

- standardizing characteristics and parameters that need to be known in the development, production and operation of supercapacitors.

Supercapacitors are used in the infra-low-frequency range; therefore, the first criterion limits the range of measuring frequencies:

$$
\begin{aligned}
& f_{\min }=0.001 \mathrm{~Hz} \quad\left(T_{\max }=\frac{1}{f_{\min }}=1000 \mathrm{~s}\right) ; \\
& f_{\max }=100 \mathrm{~Hz} \quad\left(T_{\min }=\frac{1}{f_{\max }}=0.01 \mathrm{~s}\right) .
\end{aligned}
$$


The second criterion limits the frequency sampling step $\Delta f$, which is determined by the iterative approximation. At the first stage of iterations, the measurement of the frequency dependence parameter within the selected frequency range from $0.001 \mathrm{~Hz}$ to $100 \mathrm{~Hz}$ is carried out every ten days. In this case, the grid of measuring frequencies is:

$$
f=\{0,001 ; 0,01 ; 0,1 ; 1 ; 10 ; 100\}[\mathrm{Hz}] .(16)
$$

At the next iteration steps, the change in the measured parameters $C_{i}\left(f_{i}\right)$ and $R_{i}\left(f_{i}\right)$ between adjacent frequencies is analyzed. With significant changes in these measured parameters, the frequency sampling step $\Delta f$ is reduced, and measurements are carried out between adjacent frequencies at which measurements were carried out at the previous iteration step. A significant decrease in the frequency sampling step $\Delta f$ leads to an increase in the number of $n$-links of absorption $R_{i} C_{i}$ of the mathematical model of supercapacitors, and, consequently, in the number of equations and the complexity of calculations. This leads to a significant increase in the measurement time.

If at the next iteration steps the change in the measured parameters $C_{i}\left(f_{i}\right)$ and $R_{i}\left(f_{i}\right)$ between adjacent frequencies is insignificant, then the frequency sampling step $\Delta f$ increases. In this case, the number of $n$-links of absorption $R_{i} C_{i}$ of the mathematical model decreases. This leads to improved performance of the meter.

The third criterion determines the permissible amount of change in the measured parameters $C_{i}\left(f_{i}\right)$ and $R_{i}\left(f_{i}\right)$ between adjacent frequencies. This criterion is determined by the standardizing characteristics and parameters that need to be known in the design, manufacture and operation of supercapacitors.

This completes the first stage of the iterative-recursive method. At the second stage, the numerical values $r, C_{0}, C_{i}, R_{i}$, and $R_{0}$ of the electrical parameters of the elements of the equivalent circuit of supercapacitors are determined using recursive calculations.

These recursive calculations are based on experimental studies of the parameters of supercapacitors, which show that for such capacitors the following ratios of the parameters of the equivalent circuit are observed [13]:

$$
\left\{\begin{array}{l}
r<R_{1}<R_{2}<\ldots R_{i}<\ldots<R_{n}<<R_{0} \\
\tau_{0}<\tau_{1}<\tau_{2}<\ldots<\tau_{i}<\ldots<\tau_{n}
\end{array}\right.
$$

where $\tau_{0}=r C_{0}, \tau_{1}=R_{1} C_{1}, \ldots, \tau_{\mathrm{i}}=R_{i} C_{i}, \ldots, \tau_{n}=R_{n} C_{n}$.

The formulas for calculating the capacitance $C i$ and resistance $R_{i}$ for the $i$-th absorption link are recursive, since they use the numerical values of $r, C_{0}, C_{1}, R_{1}, C_{2}, R_{2}, \ldots, C_{i-1}, R_{i-1}$, which were determined at the measuring frequencies of the selected frequency grid, respectively fmax, $f_{1}, f_{2}, \ldots f_{i-1}$ and $f_{d 1}, f_{d 2}, f_{d i-1}$, we will provide as follows:

$$
\begin{gathered}
C_{i}=\left(C_{m i}-C_{0}-\sum_{k=1}^{i-1} \frac{C_{k}}{1+4 \pi^{2} f_{i}^{2} R_{k}^{2} C_{k}^{2}}\right) \times \\
\times\left(\begin{array}{c}
f_{i}^{2}\left(C_{d i}-C_{m i}+\sum_{k=1}^{i-1} \frac{C_{k}}{1+4 \pi^{2} f_{i}^{2} R_{k}^{2} C_{k}^{2}}-\sum_{k=1}^{i-1} \frac{C_{k}}{1+4 \pi^{2} f_{d i}^{2} R_{k}^{2} C_{k}^{2}}\right) \\
f_{i}^{2}\left(C_{m i}-C_{0}-\sum_{k=1}^{i-1} \frac{C_{k}}{1+4 \pi^{2} f_{i}^{2} R_{k}^{2} C_{k}^{2}}\right)-f_{d i}^{2}\left(C_{d i}-C_{0}-\sum_{k=1}^{i-1} \frac{C_{k}}{1+4 \pi^{2} f_{d i}^{2} R_{k}^{2} C_{k}^{2}}\right)
\end{array}\right) ;
\end{gathered}
$$




$$
\begin{aligned}
& R_{i}=1 /\left(C_{m i}-\mathrm{C}_{0}-\sum_{k=1}^{i-1} \frac{C_{k}}{1+4 \pi^{2} f_{i}^{2} R_{k}^{2} C_{k}^{2}}\right) \times \\
& \left(1+\frac{f_{i}^{2}\left(C_{d \mathrm{i}}-C_{m i}+\sum_{k=1}^{i-1} \frac{C_{k}}{1+4 \pi^{2} f_{i}^{2} R_{k}^{2} C_{k}^{2}}-\sum_{k=1}^{i-1} \frac{C_{k}}{1+4 \pi^{2} f_{d i}^{2} R_{k}^{2} C_{k}^{2}}\right)}{f_{i}^{2}\left(C_{m i}-C_{0}-\sum_{k=1}^{i-1} \frac{C_{k}}{1+4 \pi^{2} f_{i}^{2} R_{k}^{2} C_{k}^{2}}\right)-f_{d i}^{2}\left(C_{d i}-C_{0}-\sum_{k=1}^{i-1} \frac{C_{k}}{1+4 \pi^{2} f_{d i}^{2} R_{k}^{2} C_{k}^{2}}\right)}\right) \times \\
& \times \sqrt{\frac{C_{\text {дi }}-C_{\mathrm{B} i}+\sum_{k=1}^{i-1} \frac{C_{k}}{1+4 \pi^{2} f_{i}^{2} R_{k}^{2} C_{k}^{2}}-\sum_{k=1}^{i-1} \frac{C_{k}}{1+4 \pi^{2} f_{d i}^{2} R_{k}^{2} C_{k}^{2}}}{4 \pi^{2}\left[f_{i}^{2}\left(C_{m i}-C_{0}-\sum_{k=1}^{i-1} \frac{C_{k}}{1+4 \pi^{2} f_{i}^{2} R_{k}^{2} C_{k}^{2}}\right)-f_{d i}^{2}\left(C_{d i}-C_{0}-\sum_{k=1}^{i-1} \frac{C_{k}}{1+4 \pi^{2} f_{d i}^{2} R_{k}^{2} C_{k}^{2}}\right)\right]}} .
\end{aligned}
$$

where $C_{m i}$ and $C_{d i}$ is the measured value of the equivalent capacitance of an extra-large capacitor at frequencies $f_{i}$ and $f_{d i}$, respectively. Unknown values $C_{i}$ та $R_{i}$ were determined from the system of equations:

$$
\left\{\begin{array}{l}
C_{m i}=C_{0}+\sum_{k=1}^{i-1} \frac{C_{k}}{1+4 \pi^{2} f_{i}^{2} R_{k}^{2} C_{k}^{2}}+\frac{C_{i}}{1+4 \pi^{2} f_{i}^{2} R_{i}^{2} C_{i}^{2}} \\
C_{d i}=C_{0}+\sum_{k=1}^{i-1} \frac{C_{k}}{1+4 \pi^{2} f_{d i}^{2} R_{k}^{2} C_{k}^{2}}+\frac{C_{i}}{1+4 \pi^{2} f_{d i}^{2} R_{i}^{2} C_{i}^{2}}
\end{array}\right.
$$

where to determine the two unknown quantities $C_{i}$ and $R_{i}$, it is necessary to measure the value of the equivalent capacitance of the capacitor at two frequencies, respectively, $f_{i}$ and $f_{d i}=f_{i-1} / 2$. Using the frequency grid, which is selected at the first iteration step, namely, the matrix row $f=\{0.001 ; 0.01 ; 0.1 ; 1,10,100\}$, allows you to obtain a five-way equivalent circuit for the supercapacitor (Fig. 3).

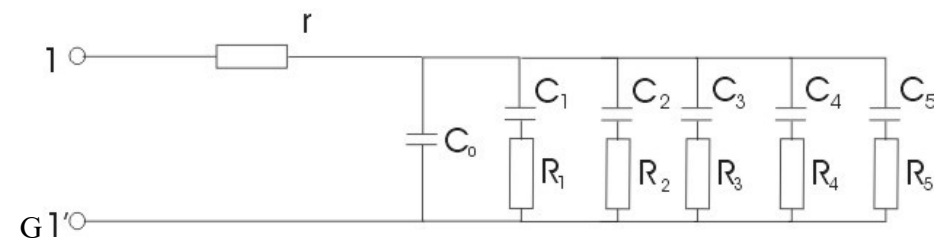

Fig. 3. An example of a five-way equivalent circuit for a supercapacitor.

Depending on the normalizing characteristics and parameters that need to be known in the development, production and operation of supercapacitors using the proposed iterativerecursive method for determining the parameters of the elements of the equivalent circuit of such objects, the number of absorption links can be increased or decreased, additional links can be introduced to improve the accuracy of calculations for the given method [6].

However, it should be noted that the introduction of additional links will lead to an increase in the measurement time of the parameters of the equivalent circuit of the supercapacitors, which is necessary for the meter of these parameters, according to the given method.

In addition, in the case of the complication of the supercapacitor equivalent circuit, one should take into account the fact that they are characterized by the presence of capacitance and active resistance of losses, which, according to a certain law, are distributed along the length or over the volume of the constructive space of the capacitor. Graphically, a fragment of the constructive space of a capacitor can be depicted using a fragment of an equivalent circuit, Fig. 4. 


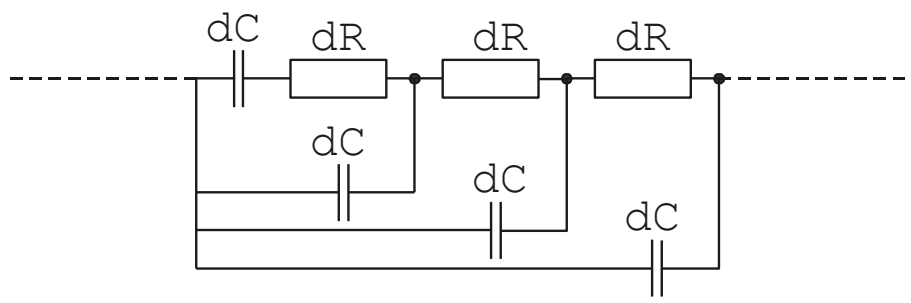

Fig. 4. Fragment of the equivalent circuit for a part of the capacitor structural space.

The structure of such an equivalent circuit includes elementary differential links of capacitance $d C$, which are charged through elementary differential links of resistances $d R$. Depending on the design of the condenser, the links $d R_{i} d C_{i}$ are distributed either along the length $\mathrm{dl}$ or over the volume $d V$ of the condenser construction space.

This approach is the most correct in the mathematical description of real capacitors. The transition from the integral characteristics of the capacitance and the active resistance of losses in the discrete links $R_{i} C_{i}$ and the equivalent circuit allows only with a certain accuracy to experimentally confirm the mathematical models of capacitors, which in general can be infinitely many.

Experimental studies carried out by the authors show that for supercapacitors the equivalent Maxwell equivalent circuit is most adequate $[5,11]$.

This conclusion can be made on the basis of the frequency dependences $C(\omega)$ and $R(\omega)$, which were experimentally obtained in the course of studying real supercapacitors.

During the measurement of the parameters of supercapacitors at low and infra-low frequencies (at $f \rightarrow 0$ ), the measured capacitance values increase. This is due to an increase in the reactance of the following links and an increase in their influence on the total result of capacitance measurement. Based on this, it is logical to assume that the representation of the model in the form of $n$-links $R_{i} C_{i}$ will depend on the number of frequencies at which the measurement is carried out. This means that for the model, the number of n-links of absorption $R_{i} C_{i}$ is, on the one hand, limited by the frequency grid of the measuring sinusoidal signal and the resolution of the measuring device in terms of capacitance $\Delta C$ and active resistance $\Delta R$. On the other hand, the number of $n$-links of the model should be limited by the requirements for standardizing characteristics and parameters that need to be known in the production and operation of supercapacitors.

\section{Conclusions}

An analysis of the results of measuring the parameters of supercapacitors showed that twoelement equivalent circuits are unsuitable for describing such objects, since there is a significant frequency dependence of the measured parameters. It is established that the parameters of supercapacitors must be diagnosed in the infra-low frequency range using a sinusoidal measuring signal. To control the parameters of supercapacitors at infra-low frequencies, direct conversion methods are most suitable, where low-resistance, highprecision, highly stable resistors are used as exemplary measures. Analysis of mathematical models and their characteristics and parameters of capacitors shows that Maxwell's mathematical model is the most adequate to real objects. Theoretical and experimental studies of the equivalent circuit of supercapacitors show that the equivalent resistance of such capacitors and their capacitance increase with decreasing frequency of the measuring signal, and the nature of these dependences is determined by the number of $n$-links of absorption and the numerical values of the elements $R_{i} C_{i}$ of each link. An iterative-recursive method for determining the parameters of the elements of the equivalent circuit of supercapacitors is 
proposed, with the help of which the number of $n$-links of absorption of the equivalent circuit and the numerical values of the elements of this equivalent circuit are determined.

\section{References}

1. T. Barić, H. Glavaš, E. K. Nyarko, Second-order supercapacitor model and its parameter extraction. International Journal of Numerical Modelling: Electronic Networks, Devices and Fields, 33(5), e2727 (2020)

2. n. Poonam, K. Sharma, A. Arora, S.K. Tripathi, Review of supercapacitors: Materials and devices. Journal of Energy Storage, 21, 801-825 (2019)

3. U. S. Sani, I. H. Shanono, A Review on Supercapacitors. Akgec International Journal of Technology (2016)

4. V. Martynyuk, M. Voynarenko, J. Boiko, O. Svistunov, Simulation of Photovoltaic System as a Tool of a State's Energy Security. International Journal of Engineering, Transactions B: Applications, 34(2), 487-492 (2021)

5. V. Martynyuk, D. Makaryshkin, J. Boyko, Frequency domain analysis for electrochemical supercapacitors, in Proceedings of the 15th IMEKO TC-4 International Symposium on Novelties in Electrical Measurement and Instrumentations, IMEKO TC4, 19-21 September 2007, Iasi, Romania, 2, 357-361 (2007)

6. V. Martynyuk, O. Eromenko, J. Boiko, T. Kałaczyński, Diagnostics of supercapacitors. MATEC Web of Conferences, 182, 01009 (2018)

7. V. Martynyuk, J. Boiko, M. Łukasiewicz, E. Kuliś, J. Musiał, (2019). Diagnostics of Solar Cells. MATEC Web of Conferences, 302, 01013 (2019)

8. J. Potencki, Modelowanie właściwości superkondensatorów. Elektronika: konstrukcje, technologie, zastosowania, 51(8), 89-93 (2010)

9. A. P. R. Fernandez, E. A. Périgo, R. N. Faria, Analytical expressions for electrochemical supercapacitor with potential dependent capacitance. Journal of Energy Storage, 44, $103156(2021)$

10. Z. Bououchma, J. Sabor, H. Aitbouh, New electrical model of supercapacitors for electric hybrid vehicle applications. Materials Today: Proceedings, 13(3), 688-697 (2019)

11. M. E. Şahİn, F. Blaabjerg, A. Sangwongwanİch, Modelling of supercapacitors based on simplified equivalent circuit. CPSS Transactions on Power Electronics and Applications, 6(1), 31-39 (2021)

12. H. Yang, Analysis of supercapacitor charge redistribution through constant power experiments, in Proceedings of the 2017 IEEE Power \& Energy Society General Meeting, 16-20 July 2017, Chicago, IL, USA, 1-5 (2017)

13. V. Martynyuk, D. Makaryshkin, J. Boyko, Electrochemical supercapacitor time domain analysis by means of multi-channel measurement system, in Proceedings of the 15th IMEKO TC-4 International Symposium on Novelties in Electrical Measurements and Instrumentations, IMEKO TC-4, 19-21 September 2007, Iasi, Romania, 1, 207-211 (2007)

14. K. Bellache, M. B. Camara and B. Dakyo, Multi-physical characterization of supercapacitor, in Proceedings of the 2017 Twelfth International Conference on Ecological Vehicles and Renewable Energies, EVER, 11-13 April 2017, Monte Carlo, Monaco, 1-5 (2017) 
15. O. Shynkaruk, J. Boiko and O. Eromenko, Measurements of the energy gin in the modified circuit signal processing unit, in Proceedings of the 2016 13th International Conference on Modern Problems of Radio Engineering, Telecommunications and Computer Science, TCSET, 23-26 Feb. 2016, Lviv, Ukraine, 582-584 (2016) 